\title{
Schamröte, Anatomie und Liebesbriefe - Jacob Henle, ein leidenschaftlicher Rationalist
}

Fritz Dross, Iris Ritzmann

Korrespondenzen:

Dr. phil. Fritz Dross Institut für Geschichte und Ethik der Medizin Glückstraße 10 D-91054 Erlangen

fritz.dross@ gesch.med.uni-erlangen.de

PD Dr. med. et lic. phil. Iris Ritzmann

Medizinhistorisches Institut der Universität Zürich Hirschengraben 82 CH-8001 Zürich

iritz@mhiz.uzh.ch
«Darwin scheint unter einer derartigen Sensibilität nicht zu leiden; er meint, dass die Menschen nicht erröthen, die im Stillen einen Fehler oder eine Sünde begangen haben». Mit diesen Worten stellte sich der Anatom und Physiologe Jacob Henle in seiner Abhandlung «Ueber das Erröthen» gegen die Ausführungen des ungleich berühmteren Charles Darwin, der wie Henle vor 200 Jahren geboren wurde. Henles Geburtstag, der 19. Juli 1809, bietet eine Gelegenheit, an diesen eigenwilligen Gelehrten zu erinnern.

Die Erklärung Darwins, diese menschliche Reaktionsweise würde durch Blicke hervorgerufen, die sich auf die betreffende Körperregion richten, leuchtete Henle nicht ein. Denn er wusste von den sensiblen Naturen, die «in der Einsamkeit, in der blossen Erinnerung an eine beschämende Situation, ihre Farbe wechseln». Autobiographische Züge lässt das angefügte Beispiel einer blamablen Erinnerung erkennen, worin ein ungenannter Jüngling «der Dame des Hauses, statt dem Dienstmädchen, ein Trinkgeld in die Hand drückte». Die eigene Emotionalität machte Henle zum Instrument, um einen Vorgang auf höchst rationale Weise zu erklären. Die enge Verflechtung von Gefühl und Verstand prägten Leben und Werk dieses eigenwilligen Wissenschaftlers, ganz besonders aber seine Zürcher Jahre.

\section{Abb. 1: Jacob Henle mit seiner ersten Frau, der Schweizerin Elise Egloff}

Jacob Henle kam in Fürth als ältestes Kind einer angesehenen jüdischen Familie zur Welt. In Fürth befand sich damals eine der bedeutendsten jüdischen Gemeinden Süddeutschlands. Im Zuge der sogenannten «Emanzipation» zog die Familie weg vom «bayerischen Jerusalem» über Mainz nach Koblenz und wählte, kurz vor Jacobs Bar-Mizwa, die Taufe, die den Aufstieg ins evangelische Bürgertum ebnete. Henle studierte in Bonn Medizin bei Johannes Müller, dem er später nach Berlin folgte und schliesslich dessen Assistent wurde. Henle verkehrte nun im Berliner Salon der Mendelssohns, wo ihm die bereits in seiner Kindheit eingetrichterten gutbürgerlichen Umgangsformen zugutekamen. Die steile Karriere knickte freilich etwas ein, als Henle wegen der Mitgliedschaft in einer Burschenschaft angeklagt wurde und nur durch die Fürsprache Alexander von Humboldts einer Inhaftierung entging.

Auch wissenschaftlich fügte sich Henle nicht stromlinienförmig in die gängigen Denkweisen ein.
Seine 1840 publizierten Überlegungen «Über Miasmen und Kontagien» legen beredtes Zeugnis von Henles methodologischem Scharfsinn und geistiger Selbstständigkeit ab. Neben parasitären, miasmatischen und unbelebt-kontagiösen Krankheitsverursachern entwickelte Henle die theoretische Möglichkeit eines «Contagium vivum» - eines lebendigen Krankheitserregers. Obwohl er dieses «Contagium vivum» nie gesehen hat, entwarf er ein Verfahren, wie man diese unentdeckten Krankheitserreger einem spezifischen Krankheitsbild zuordnen könne, und legte damit die Grundlage für die Henle-Koch'schen Postulate. Die Schrift endet in einer programmatischen Auseinandersetzung mit dem Verhältnis von Theorie und Empirie in der Medizin.

In gründlicher und vertiefender Ausarbeitung bilden diese Gedanken gleichsam das Bekenntnis zur «rationellen Medicin», wie Henle es im Eingangsartikel der gemeinsam mit dem Freund und Kollegen Karl Pfeufer in Zürich herausgegebenen «Zeitschrift für rationelle Medicin» formulierte. In Zürich wirkte Henle von 1840 bis1844 als Professor für Anatomie, bevor er nach Heidelberg und von dort 1852 nach Göttingen wechselte.

\section{Abb. 2: Die Henle'sche Schleife}

Bekannt ist Henle heute in erster Linie durch seine 1862 publizierte Arbeit «Zur Anatomie der Niere», in deren Folge der Zürcher Anatom und Physiologe Albert

\section{Abbildung 1}

Porträt des jungen Paares Elise Egloff und Jacob Henle, vermutlich um 1847. Buchcover auf der Briefedition «Elise Egloff - Die Geschichte einer Liebe in ihren Briefen» von Paula Rehberg, Zürich: Züst, 1937.

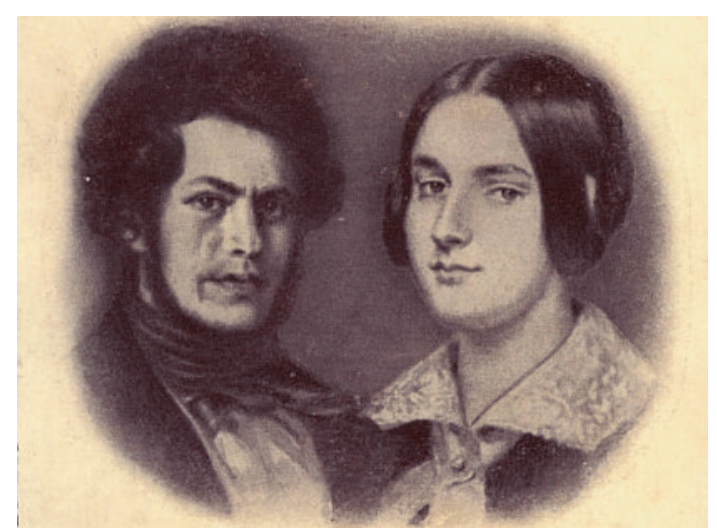


Kölliker bereits 1863 den bis heute gebräuchlichen Terminus der «Henle'schen Schleife» einführte. Die Abhandlung ist noch heute lesenswert, denn ganz unabhängig davon, ob es sich um ein Lehrbuch der Anatomie oder einen Liebesbrief handelte, pflegte Henle eine ausgesprochen literarische und emotionale Sprache, um präzise Sachverhalte zu verdeutlichen. Gefühle bildeten für ihn keinen Gegensatz zur Vernunft.

Einen Zugang zu seinen Gefühlen erlauben vor allem Henles Briefe. Die Briefwechsel mit Kollegen zeugen von seiner Eigenart, sich mit voller Leidenschaft und gleichzeitig sehr überlegt einem Gegenstand zu widmen. Tieferen Einblick in sein Innerstes gewährt der Briefwechsel mit seiner Geliebten Elise Egloff. Die junge Frau arbeitete im Haus seines Kollegen Carl Löwig, der damals ebenfalls an der Universität Zürich lehrte. Henle beschritt einen rationalen Weg, dieser Leidenschaft das Tor zu öffnen: Elise musste die gutbürgerlichen Formen gleichsam auf dem zweiten Bildungsweg in einem Internat erwerben, isoliert sowohl von ihrem Liebhaber als auch ihrer Familie und all ihren Freunden. Die ungewöhnliche Liebes- und Erziehungsgeschichte bot mehrfach zu literarischer Verarbeitung und Briefeditionen Anlass.

\section{Abb. 3: Henles Wohnung am Mühlebach in Zürich}

Über vierzig Briefe Henles an seine Geschwister und Eltern aus seiner Zürcher Zeit befinden sich noch unediert im Archiv des Medizinhistorischen Instituts der

\section{Abbildung 2}

Die Henle'sche Schleife. Erstmalig publizierte Darstellung der schleifenförmigen Kanälchen in den menschlichen Nierenpapillen. Lithografie in Jacob Henles «Zur Anatomie der Niere» Göttingen: Dieterich; 1862.

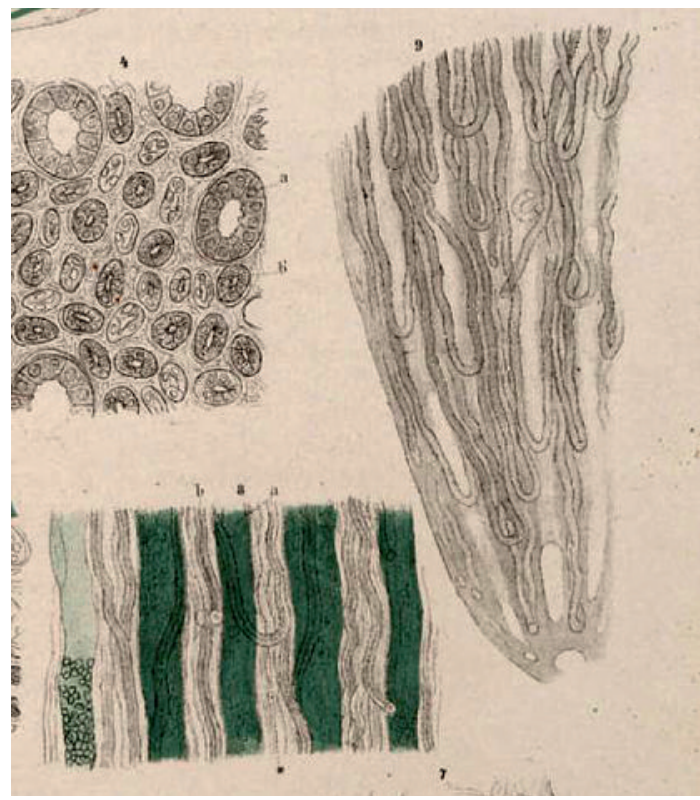

\section{Abbildung 3}

Henles Wohnung am Mühlebach in Zürich. Jacob Henle trat im Herbst 1840 die Professur für Anatomie in Zürich an. «Noch nie habe ich so schön gewohnt», schrieb er seinen Geschwistern und skizzierte den Grundriss seiner neuen Wohnung. Der Hausbesitzer habe inn zum abendlichen Musizieren mit seinen Töchtern eingeladen. Diese vier Töchter seien, so Henle, wie alle Schweizerinnen sein sollen, sehr häuslich, daneben gebildet und musikalisch.

Kein Wunder also, hat Henle sein Herz schliesslich in Zürich verloren! Medizinhistorisches Archiv, PN 199.1:2.

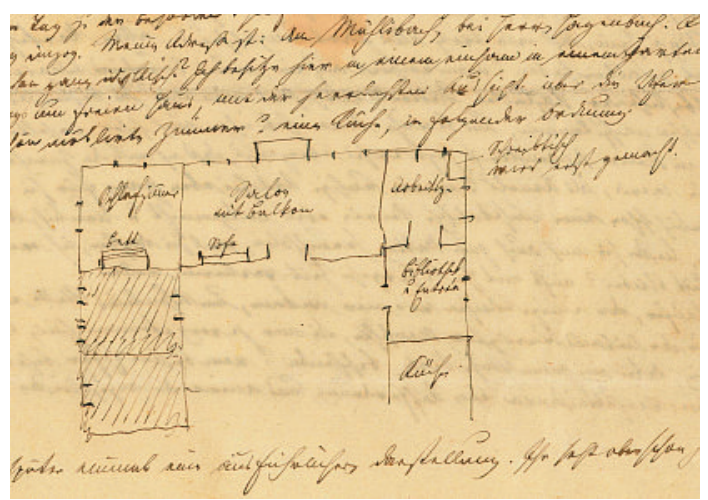

Universität Zürich. Schwärmerische Beschreibungen Zürichs und persönliche Bekenntnisse über die tiefempfundene Einsamkeit wechseln sich ab mit humorvollen Analysen seiner Kollegen und bitteren Klagen über die konservative, universitätsfeindliche Regierung. Henles Schwestern wurden offenbar auch aus der Ferne nicht müde, den Bruder zur Brautschau in den unterschiedlichsten Familien zu drängen. Henles Antworten enthalten nicht selten einen ironischen Unterton: «Tochter no 1 ausgegangen, 2 verreist, 3 Backfisch", berichtet er oder schildert - ganz der Anatom - einzelne Gesichtsregionen: «Sie hat schöne Augen und Augenbrauen, einen niedlichen Mund und weisse Zähne und macht sich daher en face ganz hübsch [...] kurz ich bin mit Eurer Wahl für mich nach erster einmaliger Anhörung zufrieden.»

Eine Arbeitsgruppe von Kolleginnen und Kollegen aus Heidelberg, Göttingen, Erlangen/Fürth und Zürich beschäftigt sich in unterschiedlicher Weise mit Jacob Henle: Das Medizinhistorische Archiv hat die Edition der erwähnten Zürcher Familienbriefe in Angriff genommen, im Foyer des Klinikums Fürth wird bis zum 10. September die Ausstellung «Bürgerliches Leben und rationelle Medicin» gezeigt, und im Zentrum Anatomie der Universitätsmedizin Göttingen kann man bis 31. Oktober die Ausstellung «Jacob Henle» besichtigen. Verschiedene Symposien und Vorträge finden im Umfeld statt, und schliesslich wird dem gebürtigen Fürther ein Band in der Schriftenreihe des Stadtarchivs Fürth gewidmet. 